\title{
Clinical and Practical Psychopharmacology \\ Antidepressant Exposure During Pregnancy any website. and Risk of Autism in the Offspring, 2: \\ Do the New Studies Add Anything New?
}

\author{
Chittaranjan Andrade, MD
}

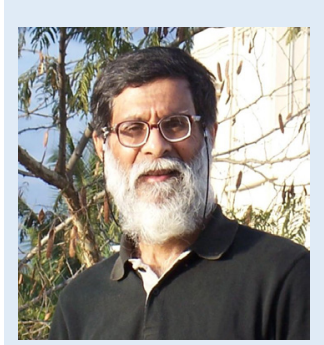

Each month in his online column, Dr Andrade considers theoretical and practical ideas in clinical psychopharmacology with a view to update the knowledge and skills of medical practitioners who treat patients with psychiatric conditions.

Department of Psychopharmacology, National Institute of Mental Health and Neurosciences, Bangalore, India (candrade@psychiatrist.com).

\section{ABSTRACT \\ Background: During the past year, at least 5 new studies, all observational in design, examined the risk of autism spectrum disorder (ASD) in children exposed to antidepressant medication in utero. These studies had not found inclusion in the many systematic reviews and meta-analyses that had also been published in the past year.}

Methods: Noteworthy methods and findings of the new studies are summarized. One of these studies is examined in detail to help the reader understand methodological and conceptual issues that are critical in the field. Some general caveats in the interpretation of the literature are also discussed.

Results: In order to reduce the limitations associated with their observational design, the new studies used many innovations, including maternal controls with mental illness, propensity score-matched controls, preconception antidepressant exposure controls, sibling controls, paternal antidepressant user controls, and modeling for the presence of an unknown confound. Two studies found an association between maternal antidepressant use during pregnancy and the risk of ASD in the offspring; these associations remained statistically significant even after covariate adjustments. The other 3 studies found that the significant association between antidepressant exposure and ASD risk was lost after statistical adjustment; that preconception antidepressant exposure was also associated with increased risk of ASD; that siblings discordant for antidepressant exposure had similar ASD risk; and that paternal antidepressant use was also associated with increased risk.

Conclusions: The new studies do not change the conclusions of the available meta-analyses. In fact, at least some of the new data strengthen the conclusion that antidepressant use during pregnancy is likely to be a marker of more severe illness and that inadequately measured, unmeasured, or unknown genetic, behavioral, and environmental confounds associated with more severe illness (rather than the antidepressant exposure by itself) may be responsible for the increased risk of ASD.

J Clin Psychiatry 2017;78(8):e1052-e1056

https://doi.org/10.4088/JCP.17f11916

(c) Copyright 2017 Physicians Postgraduate Press, Inc.

\section{Introduction}

During the past 2 decades, more than a dozen cohort and case-control studies have examined the association between maternal antidepressant exposure during pregnancy and the risk of autism spectrum disorder (ASD) in the offspring. In approximately the last year alone, at least 6 systematic reviews and meta-analyses ${ }^{1-6}$ with different objectives and different methods examined the subject; the findings of these meta-analyses were reasonably consistent ${ }^{7}$ and are summarized in Table 1. The general consensus appears to be that confounding by indication may explain the increased risk of ASD that is associated with gestational exposure to antidepressant medication.

At least 5 new studies ${ }^{8-12}$ have been published this year, none of which had been included in the meta-analyses. ${ }^{1-6}$ The present article examines these studies to determine whether these new studies change the meta-analysis findings and conclusions that are presented in Table 1. By examining one of these 5 new studies in detail, the present article also seeks to help the reader obtain a critical view of the field.

\section{Caveats}

There are 4 important caveats, concerning the studies in the field, of which readers need to be aware. First, it may seem that if an impressive number of studies consistently find that antidepressant exposure during pregnancy is associated with an increased risk of ASD in the offspring, then the finding must be true. This is undeniably so. What is fallacious is the continuation of thought that if antidepressants are consistently associated with an increased risk, then antidepressants must be responsible for the risk; the fallacy, of course, is that the same confounds, all related to confounding by indication, may operate in all the studies. These confounds are the genetic and behavioral accompaniments of maternal mental illness for which the antidepressants were prescribed.

There are no randomized controlled trials (RCTs) of the safety of antidepressant drugs during pregnancy; all the studies in the field are observational in nature. Many ingenious methods have been devised to try to get around the problem of confounding by indication. These include, for example, using propensity-matched controls and sibling controls, but neither procedure eliminates residual confounding arising from incompletely measured, unmeasured, and unknown confounds. ${ }^{13,14}$

Second, it may seem that there is an impressively large number of original studies on the subject, but several of the studies actually examined more or less the same population. 


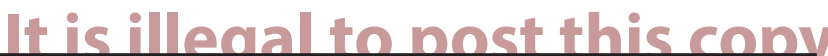
Table 1. General Conclusions of Meta-Analyses of Studies of the Risk of ASD Following Gestational Exposure to Antidepressant Drugs ${ }^{7}$

1. There is an increased risk of ASD in children exposed to antidepressant drugs in utero.

2. The risk is reduced after adjusting for confounding variables and may no longer be statistically significant after adjusting for maternal psychiatric illness.

3. Antidepressant exposure is associated with an increased risk of ASD in the offspring even when the exposure is limited to the preconception period, when there is no way in which the drugs can have a physiological effect on the fetus.

4. Putting these findings together, it appears that maternal mental illness may explain much or all of the risk of ASD associated with antidepressant exposure during pregnancy.

Abbreviation: $\mathrm{ASD}=$ autism spectrum disorder.

So, the effective database supporting an association between antidepressant exposure and ASD is actually smaller than it seems. For example, Hviid et $\mathrm{al}^{15}$ examined all live births in Denmark between January 1996 and December 2005, and Gidaya et $\mathrm{al}^{16}$ examined all children born in Denmark between January 1997 and December 2006. However, the former group used the data in a cohort study design, whereas the latter group used the data in a case-control study design. Therefore, there was substantial overlap in the source data, and only the method of examination of the data differed. This overlap of data was recognized by the authors of many meta-analyses, and studies with overlapping data were either omitted from meta-analysis or examined separately and not together. ${ }^{7}$ There is almost certainly some overlap in the newer studies, as well, as readers will discern from the information on the geographical and temporal sources of the data.

Third, studies that looked at the same data in different ways arrived at different conclusions. For example, one study that examined Danish registry data concluded that there was no association between maternal use of selective serotonin reuptake inhibitors (SSRIs) during pregnancy and ASD in the offspring ${ }^{15}$; another study, looking at almost the same data but with a different study design and with different covariates, found that in utero exposure to SSRIs was associated with an increased risk of ASD in the offspring. ${ }^{16}$ This is a little like the story of the blind men and the elephant; the outcome depends on how the data are examined, regardless of what the true nature of the data is.

Finally, some studies drew conclusions that were at variance with what their findings actually suggested. For example, whereas Gidaya et $\mathrm{al}^{16}$ found an association between in utero exposure to SSRIs and ASD risk in the offspring, they also found an almost identical association between exclusive preconception exposure and ASD risk. Furthermore, the association between preconception exposure and ASD risk remained statistically significant even when the duration of exposure was brief. Logically, one should conclude that preconception exposure cannot affect the fetus, and so it must be the indication for which the antidepressants were prescribed that increases the ASD risk and not the antidepressant treatment itself. Nevertheless,

\section{Table 2. Important Findings From the Stockholm Youth} Cohort Data ${ }^{8}$

1. Relative to exposure to maternal psychiatric disorder untreated with antidepressants, gestational exposure to antidepressant medication was associated with an increased risk of ASD in the offspring (OR $=1.45 ; 95 \%$ $\mathrm{Cl}, 1.13-1.85)$. When further examined, the finding remained significant for ASD without intellectual diability $(\mathrm{OR}=1.57 ; 95 \% \mathrm{Cl}, 1.21-2.04)$ but not for ASD with intellectual disability $(\mathrm{OR}=0.72 ; 95 \% \mathrm{Cl}, 0.38-1.77)$.

2. Assuming that the finding described above was causal and not driven by confounding by indication, the population-attributable risk of antidepressant-related ASD was estimated to be $2.1 \%(95 \% \mathrm{Cl},-0.7 \%$ to $4.7 \%)$.

3. In a propensity-matched subset, the association between antidepressant exposure and ASD was significant for ASD as a whole $(\mathrm{OR}=1.68 ; 95 \% \mathrm{Cl}, 1.23-2.30)$ and specifically for ASD without intellectual disability $(\mathrm{OR}=1.76 ; 95 \% \mathrm{Cl}, 1.26-2.46)$ but not for ASD with intellectual disability $(\mathrm{OR}=1.25 ; 95 \% \mathrm{Cl}, 0.52-3.03)$.

4. In a sibling-matched analysis, the association between antidepressant exposure and ASD was not significant for ASD as a whole $(O R=1.36$; $95 \% \mathrm{Cl}, 0.84-2.20)$ or for ASD with $(\mathrm{OR}=1.57 ; 95 \% \mathrm{Cl}, 0.92-2.66)$ or without $(\mathrm{OR}=0.78 ; 95 \% \mathrm{Cl}, 0.24-2.54)$ intellectual disability.

5. Paternal antidepressant use was not significantly associated with ASD risk for $\mathrm{ASD}$ as a whole $(\mathrm{OR}=1.13 ; 95 \% \mathrm{Cl}, 0.68-1.88)$ or for $\mathrm{ASD}$ with $(\mathrm{OR}=1.18 ; 95 \% \mathrm{Cl}, 0.68-2.08)$ or without $(\mathrm{OR}=0.91 ; 95 \% \mathrm{Cl}, 0.26-3.27)$ intellectual disability.

Abbreviations: $\mathrm{ASD}=$ autism spectrum disorder, $\mathrm{Cl}=$ confidence interval, $\mathrm{OR}=$ odds ratio.

the authors ${ }^{16}$ ignored the obvious and suggested that antidepressants play a causal role in the adverse outcome.

Other commentaries on the literature in the field have also been published. ${ }^{17-20}$

\section{Rai et al}

Findings. This article ${ }^{8}$ is examined in some detail because it received disproportionate attention in the scientific and lay press. The authors ${ }^{8}$ described an observational prospective cohort study of 254,610 children and adolescents, aged 4-17 years, living in Stockholm County, Sweden, during 20012011. The data were drawn from the Stockholm Youth Cohort intergenerational linkage study $(n=735,096)$, supported by relevant medical registers. Medication use during pregnancy was ascertained at a median of 10 weeks of gestation and was defined as a prescription for an antidepressant drug between 30 days before the start of pregnancy and the date of birth.

There were 3,342 children identified with exposure to antidepressants during pregnancy; 136 (4.1\%) had been diagnosed with ASD. There were 12,325 children whose mothers had a psychiatric disorder but who had not had gestational exposure to an antidepressant; of these, 353 (2.9\%) had been diagnosed with ASD. In the rest of the cohort, 238,943 children had no exposure to either maternal psychiatric disorder or antidepressants, and 4,889 (2.1\%) of these had been diagnosed with ASD. Important findings from this study are presented in Table 2 .

In summary, the authors ${ }^{8}$ found that even after adjusting for a wide range of potential confounds, antidepressant exposure was associated with an increased risk of ASD over and above that associated with maternal mental illness; the risk remained significant in a propensity-matched subset; and the risks were significant only for ASD without 


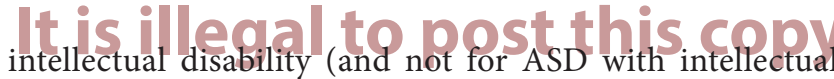

disability). Supporting the association between antidepressant exposure and ASD risk was the finding that paternal use of antidepressants was not associated with increased ASD risk; this diminishes the probability that family and environmental confounds explain the risk of ASD in the offspring. In contrast, the risk was not significantly different in siblings discordant for antidepressant exposure, negating the conjecture that antidepressant exposure causally explains the risk.

The authors ${ }^{8}$ noted that their simulations for the potential impact of unmeasured confounding indicated that the unmeasured confound, if it exists, would need to be a strong risk factor for ASD. However, they also observed that statistical significance was obtained only for ASD without intellectual disability, a phenotype that may be more heritable, suggesting that unmeasured genetic confounds may explain the ASD risk. Finally, an important finding was that if antidepressant exposure was causal for ASD risk, then avoiding ASD exposure during pregnancy would prevent only about $2 \%$ of the cases of ASD in this population.

Most but not all of the findings of this study ${ }^{8}$ appeared to implicate antidepressant exposure as a mediator of the ASD risk.

Comments. This study had several important strengths. The authors ${ }^{8}$ compared mothers who used antidepressants during pregnancy with mothers who had a psychiatric diagnosis but who did not use antidepressants; adjustments were made for individual psychiatric diagnoses in secondary analyses. Because the severity of depression was not measured, they attempted to control for severity through the use of many different cross-sectional and longitudinal proxies. They performed propensity-matched analyses. They performed (discordant) sibling-matched analyses, which indirectly control for shared unknown and unmeasured genetic and environmental confounds. They performed negative control analyses in which risks were examined in association with paternal antidepressant use during the pregnancy. Finally, they used statistical methods to estimate the effect of an unknown binary confound.

On the flip side, because their study was not an RCT, limitations of the observational design are many. There is no assurance that the confounds were accurately measured and recorded in the databases or that the proxies were sufficiently valid or complete representations of what they were intended to represent. The adjustments for known and measured confounds can never compensate for the unadjusted effects of poorly measured, known but unmeasured, and unknown and unmeasured confounds; these comments apply to propensity-matched and other analyses, as well. ${ }^{12,13}$ The sibling-matched analysis only partially controls for genetic and environmental confounds. This is because sibs have similar but not the same DNA, and sibs have similar but not the same material and emotional environment. Thus, there is plenty of room for differences and hence for epigenetic effects. ${ }^{13}$ The depressed father, negative control analysis is like the sibling-matched analysis because it helps control for shared genetic and environmental confounds, but the

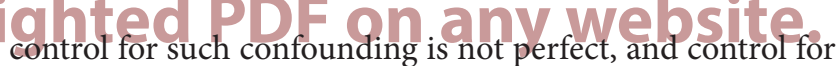
confounding by indication is not complete. Importantly, because of the nature of their data, the authors could not perform what might have been the most important controlled analysis of all: the ascertainment of risk in women who discontinued antidepressants before pregnancy. If this analysis had been possible, and if the results had been found to be significant as reported by many meta-analyses (Table 1), then the meaningfulness of the statistical significance of the remaining analyses would diminish. The lack of significance of the discordant sibling analysis is itself a noteworthy point.

Therefore, on the basis of the findings of this study, all that can be concluded with confidence is that women who need/use an antidepressant during pregnancy may be slightly more at risk of having an autistic child. The drug exposure could merely be a marker; causality cannot be inferred. More importantly, if depression severe enough to necessitate antidepressant use is the causal factor (through alcohol/substance use, abnormal eating behavior, neglect of self-care, neglect of medical guidance during pregnancy, or exposure to other risk factors), then antidepressant use may actually decrease the risk of autism through effectively treating the depression and reducing the exposure to these risk factors. However, in the absence of an RCT design, it is impossible to control for severity of depression and for other poorly measured, unmeasured, and unknown confounds.

\section{Sujan et al}

These authors ${ }^{9}$ described a retrospective cohort study of Swedish offspring who were born between 1996 and 2012 and followed through 2013. The data were extracted from relevant Swedish registries. There were 1,580,629 children in all, born to 943,776 mothers. ASD was diagnosed in 299 (5.28\%) of antidepressant-exposed children as compared with $14,318(2.14 \%)$ of unexposed children (hazard ratio $[\mathrm{HR}]=1.64 ; 95 \%$ confidence interval $[\mathrm{CI}], 1.46-1.83)$. However, the risk was not significantly different in siblings who were discordant for antidepressant exposure $(\mathrm{HR}=0.83$; 95\% CI, 0.62-1.13). The risk was elevated in exposure that was limited to the pre-pregnancy period, defined as 3-9 months before conception ( $\mathrm{HR}=1.40 ; 95 \% \mathrm{CI}, 1.02-1.93)$ as well as after first trimester exposure $(\mathrm{HR}=1.75 ; 95 \% \mathrm{CI}, 1.49-2.07)$. Finally, paternal antidepressant use during the first trimester was also associated with a significantly elevated risk of ASD in the offspring $(\mathrm{HR}=1.31 ; 95 \% \mathrm{CI}, 1.05-1.62)$. The results were similar in analyses restricted to SSRI exposure. The authors ${ }^{9}$ concluded that, after accounting for confounding variables, first trimester exposure to antidepressant drugs, relative to no exposure, was not associated with an increased risk of ASD.

This study provides strong evidence for confounding by indication. Because there is no physiological way in which pre-pregnancy antidepressant use or paternal antidepressant use can directly influence the risk of ASD in the offspring, factors other than antidepressant exposure during pregnancy must be linked to the risk. These factors could be maternal mental illness and accompaniments thereof, environmental 
factors, and others. Such an interpretation also explains why the risk of ASD did not differ significantly between offspring discordant for antidepressant exposure.

\section{Brown et al}

These authors ${ }^{10}$ described a retrospective cohort study of serotonin reuptake inhibitor (SSRI or serotoninnorepinephrine reuptake inhibitor [SNRI]) antidepressant exposure during pregnancy, drawing data from health administration databases in Ontario, Canada. The sample included 35,906 singleton births. The incidence of ASD was 4.51 vs 2.03 per 1,000 person-years in antidepressant exposed vs unexposed offspring $(\mathrm{HR}=1.59 ; 95 \% \mathrm{CI}$, 1.17-2.17). After inverse probability of treatment weighting based on a high-dimensional propensity score, the finding was no longer statistically significant $(\mathrm{HR}=1.61 ; 95 \% \mathrm{CI}$, 0.997-2.59). Importantly, antidepressant exposure 3-12 months before pregnancy was associated with an increased risk of ASD in the offspring $(\mathrm{HR}=1.85 ; 95 \% \mathrm{CI}, 1.37-2.51)$. Also importantly, there was no difference in ASD risk in antidepressant-exposed vs unexposed sibs (3.40 vs 2.05 per 1,000 person-years; $\mathrm{HR}=1.60 ; 95 \% \mathrm{CI}, 0.69-3.74)$.

In other analyses, first trimester exposure and second/ third trimester exposure were both associated with increased risk, but not in the weighted analyses. SSRIs and SNRIs were also each associated with increased ASD risk but, again, not in the weighted analyses.

Thus, this study found an association between antidepressant exposure during pregnancy and ASD risk in the offspring; however, after weighted matching, the risk was no longer statistically significant. Furthermore, when siblings discordant for SSRI exposure were compared, the risk was not significant, suggesting that shared maternal, family, and/or environmental variables, rather than antidepressant exposure, explained the risk. Finally, exposure limited to the pre-pregnancy period was also associated with an increased ASD risk, suggesting that antidepressant use is a marker of risk rather than a cause. The authors ${ }^{10}$ concluded that in utero antidepressant exposure, relative to no exposure, was not associated with ASD risk in the child.

\section{Liu et al}

These authors ${ }^{11}$ described a population-based cohort study of the risk of psychiatric disorders after antidepressant use during pregnancy. They extracted data from Danish registers and identified 905,383 singleton live births. In the only analysis relevant to the present review, the authors found that, relative to women who discontinued antidepressants during pregnancy, those who continued antidepressants were more likely to have a child who developed ASD $(\mathrm{HR}=1.23$; 95\% CI, 1.01-1.51). They could not rule out the possibility that continuation of antidepressants was a marker for more severe illness. They therefore concluded that the association between antidepressant exposure in utero and the increased risk of psychiatric disorders in the offspring may be attributable to the severity of maternal mental illness in combination with antidepressant drugs. They acknowledged

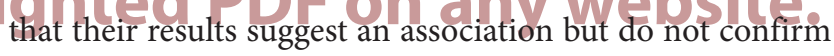
causality.

\section{Viktorin et al}

These authors ${ }^{12}$ used Swedish registers to identify all children born during 2006 and $2007(n=179,007)$ and followed through 2014; there were 1,641 (0.9\%) children with a diagnosis of ASD. Relative to unexposed children, the risk of ASD was elevated in children who had been exposed to antidepressants during pregnancy (relative risk $[\mathrm{RR}]=2.46$; 95\% CI, 1.97-3.05), but this risk was no longer statistically significant after adjustment for potential confounders $(\mathrm{RR}=1.23$; 95\% CI, 0.96-1.57). In a subsample of children $(\mathrm{n}=18,551)$ whose mothers had a lifetime diagnosis of anxiety or depression, neither crude $(\mathrm{RR}=1.30$; 95\% CI, $0.99-1.71)$ nor adjusted $(\mathrm{RR}=1.07 ; 95 \% \mathrm{CI}, 0.80-1.43)$ risks were statistically significant. The authors ${ }^{12}$ presented a large number of additional analyses, but these were all exploratory in nature or served as sensitivity analyses, and they did not change the overall findings, especially because none of the significant findings remained significant after correction of the $P$ value for multiple hypothesis testing.

An important strength of this study is that antidepressant exposure in the main analysis was defined as the issue of at least 2 prescriptions that overlapped pregnancy; this increases the likelihood that the medications were actually being taken. The authors concluded that antidepressant exposure during pregnancy does not appear to be causal in increasing the ASD risk in the offspring; rather, the observed associations may be due to factors related to maternal mental illness. They considered that, on the basis of their findings, the risk of ASD should not be a consideration to withhold antidepressant treatment if indicated during pregnancy. ${ }^{12}$

\section{Synthesis and Take-Home Message}

Two studies ${ }^{9,10}$ rejected an association between antidepressant exposure and ASD risk, and 3 studies ${ }^{8,11,12}$ suggested that an association exists but that causality is unproven. Thus, in support of the findings of the metaanalyses (Table 1), there is a growing body of evidence that invokes confounding by indication and discredits a causal association between gestational antidepressant exposure and ASD risk. At the risk of sounding repetitive, it is asserted that all the studies in the field, by virtue of their observational design, do not and cannot identify a causal role for antidepressants because, novel attempts to deal with confounding by indication notwithstanding, they cannot account for incompletely measured, unmeasured, and unknown genetic, environmental, behavioral, and other confounds.

The field will not be advanced by the conduct and publication of more of such observational studies because their findings can never be conclusive. As one commentary appropriately observed, it is time to move away from the focus on antidepressant medications alone and examine whether some mothers and their offspring benefit from prenatal maternal antidepressant treatment. ${ }^{20}$ 
1. Kobayashi T, Matsuyama T, Takeuchi M, et al. Austism spectrum disorder and prenatal exposure to selective serotonin reuptake inhibitors: a systematic review and metaanalysis. Reprod Toxicol. 2016;65:170-178.

2. Kaplan YC, Keskin-Arslan E, Acar S, et al. Prenatal selective serotonin reuptake inhibitor use and the risk of autism spectrum disorder in children: a systematic review and metaanalysis. Reprod Toxicol. 2016;66:31-43.

3. Brown HK, Hussain-Shamsy N, Lunsky Y, et al. The association between antenatal exposure to selective serotonin reuptake inhibitors and autism: a systematic review and meta-analysis. J Clin Psychiatry. 2017;78(1):e48-e58.

4. Mezzacappa A, Lasica PA, Gianfagna F, et al. Risk for autism spectrum disorders according to period of prenatal antidepressant exposure: a systematic review and meta-analysis. JAMA Pediatr. 2017;171(6):555-563.

5. Kaplan YC, Keskin-Arslan E, Acar S, et al. Maternal SSRI discontinuation, use, psychiatric disorder and the risk of autism in children: a meta-analysis of cohort studies [published online ahead or print July 21, 2017]. Br J Clin Pharmacol.

6. Andalib S, Emamhadi M, Yousefzadeh-Chabok $\mathrm{S}$, et al. Maternal SSRI exposure increases the risk of autistic offspring: a meta-analysis and systematic review. Eur Psychiatry. 2017. pregnancy and risk of autism in the offspring: meta-review of meta-analyses. J Clin Psychiatry. 2017;78(8):e1047-e1051.

8. Rai D, Lee BK, Dalman C, et al. Antidepressants during pregnancy and autism in offspring: population based cohort study. BMJ. 2017;358:j2811.

9. Sujan AC, Rickert ME, Oberg AS, et al. Associations of maternal antidepressant use during the first trimester of pregnancy with preterm birth, small for gestational age, autism spectrum disorder, and attention-deficit/ hyperactivity disorder in offspring. JAMA. 2017;317(15):1553-1562.

10. Brown HK, Ray JG, Wilton AS, et al. Association between serotonergic antidepressant use during pregnancy and autism spectrum disorder in children. JAMA. 2017;317(15):1544-1552.

11. Liu $X$, Agerbo E, Ingstrup KG, et al. Antidepressant use during pregnancy and psychiatric disorders in offspring: Danish nationwide register based cohort study. BMJ. 2017;358:j3668.

12. Viktorin A, Uher R, Reichenberg A, et al. Autism risk following antidepressant medication during pregnancy [published online ahead of print May 22, 2017]. Psychol Med.

13. Andrade C. Propensity score matching in nonrandomized studies: a concept simply explained using antidepressant treatment during pregnancy as an example. J Clin Psychiatry. 2017;78(2):e162-e165.

14. Andrade C. Offspring outcomes in studies of antidepressant-treated pregnancies depend on the choice of control group. J Clin Psychiatry. 2017;78(3):e294-e297.

15. Hviid A, Melbye M, Pasternak B. Use of selective serotonin reuptake inhibitors during pregnancy and risk of autism. NEngl J Med. 2013;369(25):2406-2415.

16. Gidaya NB, Lee BK, Burstyn I, et al. In utero exposure to selective serotonin reuptake inhibitors and risk for autism spectrum disorder. J Autism Dev Disord. 2014;44(10):2558-2567.

17. Andrade $C$. Antidepressant use in pregnancy and risk of autism spectrum disorders: a critical examination of the evidence. J Clin Psychiatry. 2013;74(9):940-941.

18. Andrade $C$. Adverse outcomes following serotonin reuptake inhibitor exposure during pregnancy. J Clin Psychiatry. 2016;77(2):e199e200.

19. King BH. Association between maternal use of SSRI medications and autism in their children. JAMA. 2017;317(15):1568-1569.

20. Oberlander TF, Zwaigenbaum L. Disentangling maternal depression and antidepressant use during pregnancy as risks for autism in children. JAMA. 2017;317(15):1533-1534. 